Voix et Images

\title{
Guy Delahaye et la modernité littéraire ou la revanche des " exotistes "
}

\section{Annette Hayward}

Volume 13, numéro 2 (38), hiver 1988

Le propre du corps Roger Des Roches

URI : https://id.erudit.org/iderudit/200714ar

DOI : https://doi.org/10.7202/200714ar

Aller au sommaire du numéro

Éditeur(s)

Université du Québec à Montréal

ISSN

0318-9201 (imprimé)

1705-933X (numérique)

Découvrir la revue

Citer cet article

Hayward, A. (1988). Guy Delahaye et la modernité littéraire ou la revanche des " exotistes ». Voix et Images, 13(2), 326-329. https://doi.org/10.7202/200714ar d'utilisation que vous pouvez consulter en ligne.

https://apropos.erudit.org/fr/usagers/politique-dutilisation/ 


\section{Essai}

\section{Guy Delahaye et la modernité littéraire ou la revanche des «exotistes»}

\section{par Annette Hayward, Université Queen's}

En voulant restituer au poète Guy Delahaye la place qui lui revient dans l'histoire de la littérature québécoise, le récent livre de Robert Lahaise, Guy Delahaye et la modernité littéraire ${ }^{1}$, constitue lui-même une nouvelle étape dans la querelle des régionalistes et des «exotistes» 2 . Quant au créateur de Guy Delahaye, Guillaume Lahaise, qui occupe plus de la moitié du volume, son fils lui pardonne difficilement, semble-t-il, d'avoir imposé silence à ce poète si original. Au fur et à mesure que le blâme de cet assassinat se déplace vers les «terroiristes», cependant, cette attitude s'adoucit sensiblement.

Un parti pris voulu et éloquent, donc, en faveur de Guy Delahaye et ses amis, qui renvoie subtilement aux enfers, à force de ridicule, les adeptes du régionalisme, Albert Lozeau (critique) et même l'École littéraire de Montréal. Cela dit, il s'agit d'un livre fascinant et propre à intéresser non seulement les littéraires, mais également les historiens et les sociologues. L'auteur écrit dans un style alerte, plein de verve et d'humour un peu mordant. Le ton et les procédés utilisés, surtout lorsqu'il parle des attaques contre Guy Delahaye, ne sont d'ailleurs pas sans liens avec le discours autrefois adopté par les meilleures plumes de la querelle entre les régionalistes et les «exotistes». Ainsi, il donnera en exemple les extraits les plus ridicules qui soient imaginables de Blanche Lamontagne et de Lionel Groulx, répétera à plusieurs reprises les textes les plus élogieux sur Guy Delahaye, et laissera souvent transparaître sa propre nature passionnée. Cela présente l'avantage de bien rendre la saveur et l'ambiance de l'époque.

En tant que fils du poète, Robert Lahaise dispose d'une documentation privilégiée (poèmes inédits, réflexions et notes, agendas, correspondance, coupures de journaux, arbre généalogique, photos, connaissances intimes sur la vie familiale) qui lui permet de livrer une foule de renseignements nouveaux sur tout ce qui touche - de près ou de loin - à la vie et à l'œuvre de son père. À cela s'ajoute, de toute évidence, une recherche personnelle de longue haleine. D'aucuns pourraient lui reprocher d'avoir donné trop de détails sur la famille, mais il est vraiment fascinant de pouvoir suivre ainsi cet être d'exception à partir de (et même avant) sa naissance dans ce lieu privilégié des dieux qu'est le mont SaintHilaire, jusqu'à son fidèle retour à Saint-Jean-de-Dieu pour y mourir, à l'âge de 81 ans. L'auteur, heureusement pour nous, n'a pas hérité de la discrétion extrême qui caractérisait son père. 
Le gros volume qui en résulte se divise en quatre parties d'environ une centaine de pages chacune: «Une jeunesse bourgeoise, 1888-1910»; «Delahaye le moderniste» (sur les deux recueils de Guy Delahaye parus en 1910-1912, mais que le biographe ne semble pas vouloir limiter en assignant des dates); «le Médecin de l'errance (1912-1924)»; le Psychiatre qui s'exile (1924-1969)». Il s'en dégage le portrait d'un jeune homme individualiste et cultivé, tôt porté vers le mysticisme et l'angoisse existentielle, d'une fidélité extrême envers les êtres qu'il aime. Après le scandale provoqué par les Phases et Mignonne..., la recherche poétique cède peu à peu la place chez lui à la préoccupation religieuse. Cette tendance se trouve encouragée par son obsession de la mort, déjà évidente dans les Phases, mais exacerbée par la typhoïde dont il a failli mourir à Paris et par le décès de sa mère. Sa santé délicate et son insatisfaction, sa quête intérieure, sont à l'origine des fréquents séjours à l'étranger (Paris, la Californie, Cuba, la Floride) entre 1912 et 1924, auxquels mettra fin son engagement comme psychiatre à l'hôpital Saint-Jean-de Dieu, poste qu'il occupera pendant trente-quatre ans. La rencontre de sa future femme, leur mariage et l'arrivée des enfants ne mettront qu'un frein temporaire et tout relatif aux préoccupations religieuses qui occuperont par la suite une place de plus en plus grande dans sa vie. Il publiera d'ailleurs, en 1934, un chemin de la croix anonyme intitulé l'Unique voie à l'unique But. Il faut dire que Guillaume Lahaise, en dépit de sa douceur et de sa grande timidité, était un extrémiste dans le vrai sens du terme et ne faisait jamais les choses à moitié, en particulier lorsqu'il s'agissait de poésie ou de religion. Il appert, en lisant ce volume, que les tendances mystiques du poète, probablement en grande partie responsables de l'originalité de son œuvre, se trouveraient également à l'origine de son silence. La littérature de la modernité, comme en témoigne l'expérience de Nelligan, est une véritable religion, fort exigeante. Mais elle ne saurait cœxister avec le Dieu jaloux et toutpuissant auquel Guillaume Lahaise choisit finalement de se soumettre 3 .

Dès l'âge de dix ans, Guillaume Lahaise s'éveillait à l'art en [...] regardant peindre [Ozias] Leduc ( p. 428) qui deviendra par la suite, semble-t-il, son père spirituel. À seize ans, il connaîtra le coup de foudre en lisant Émile Nelligan, poète qui demeurera pour lui, jusqu'à la fin de sa vie, un dieu (p. 452).

C'est à l'Université Laval de Montréal que se formera ce groupe d'amis que Robert Lahaise désigne comme l'indissoluble quatuor des rexotistes» (p. 61): Paul Morin, Marcel Dugas, René Chopin et Guy Delahaye.. À ce propos, la section sur les quatre cavaliers de l'Apocalypse (p. 60-75) est particulièrement précieuse, à notre avis, par l'aperçu succinct et lucide qu'elle donne de la destinée des trois camarades de Guy Delahaye, ces «choyés» des dieux qui promettaient tant... Ce livre réussit également à résoudre enfin l'énigme de «l'Encéphale», société secrète fondée par Guy Delahaye 4 .

Après avoir désigné, de façon un peu simpliste, un article d'Adrien Plouffe contre Guy Delahaye comme le Début de la «Querelle des Anciens et des Modernes» (voir «Chronologie») 5 , Robert Lahaise mentionne un différend semblable qui aura lieu un an plus tard dans le Nationaliste, alors que Paul Morin 
se trouve parodié par «Pauline Morinette», défendu avec passion par René Chopin et réattaqué par Pauline et «Renée». L'hypothèse selon laquelle Pauline et Renée seraient peut-être l'abbé J.-M. Melançon et Albert Lozeau qui, vers la même époque, s'étaient moqués des «terroiristes» sous les pseudonymes de Luc et Ch. Launier, nous semble peu plausible. D'abord, Pauline et Renée, au lieu de se montrer complices, se contredisent ${ }^{6}$. De plus, la féminisation des noms de Morin et de Chopin, qui laisse sous-entendre qu'une fréquentation trop poussée de la culture française entraînerait une certaine efféminisation, ne correspond guère, à notre connaissance, à l'attitude d'Albert Lozeau et de Lucien Rainier envers la France.

Les propos du biographe-historien sur l'École littéraire de Montréal et en particulier sur son refus de la candidature de Guy Delahaye nous semblent également sujets à caution7. Son désir de venger le traitement injuste des historiens de la littérature envers Guy Delahaye et ses amis l'entraîne à contester le rôle joué par ce cénacle.

Si l'auteur a raison d'insister sur la modernité de l'œuvre de Delahaye, le fait d'envisager tant d'événements de cette époque de l'unique point de vue de leur lien avec ce poète fausse quelque peu le tableau qui s'en dégage. Le lecteur en sort avec l'impression que cette «Querelle des Anciens et des Modernes», simple répétition du conflit français du XVIIe siècle, consiste en l'opposition stupide et réactionnaire d'une foule de «terroiristes» au modernisme de «lindissoluble quatuor des "exotistes"» formé par Delahaye (le plus moderne et donc le plus important?) et ses amis. Le Nigog et Victor Barbeau auraient essayé de rectifier la situation mais, semble-t-il, en vain.

Une telle perspective résulte du fait d'envisager ce conflit uniquement du point de vue des œuvres littéraires. Mais même là, nous serions tentée de donner raison à Jean Éthier-Blais qui, dans le Devoir du 12 septembre 1987, affirme que la modernité n'est pas en soi une garantie de qualité et que la valeur intrinsèque de la poésie de Paul Morin l'emporte quand même sur celle, certes très valable, de Guy Delahaye. Mais comment en être certain, lorsque cette poésie est aujourd'hui quasiment introuvable (à quand donc l'édition critique de cette œuvre?).

Si la querelle entre les régionalistes et les «exotistes» peut paraître ridicule et insensée à cette époque lorsqu'on la regarde du point de vue des œuvres ou comme une répétition - tardive! — d'un vieux conflit européen, tel n'est pas le cas si on l'examine dans la perspective de l'institution littéraire. Elle apparaît alors comme un phénomène spécifiquement québécois, un brassage explosif et révélateur d'idées fondamentales à la problématique de cette littérature et (entre autres) de son rapport avec la littérature française. Il s'agit d'une querelle idéologique qui tournait moins autour de la question de la qualité des cuvres que de celle d'une possible «autonomie». Et sans vouloir minimiser l'importance du désir de liberté, d'universalisme et de modernisme chez les «exotistes» (qui, à notre sens, étaient bien plus que quatre), précisons que le réveil littéraire des 
années 60 et 70 avait vu revenir des préoccupations et des arguments qui rappelaient étrangement certains propos des régionalistes au début du siècle ${ }^{8}$. Mais depuis, on le sait, le paysage s'est encore modifié: l'heure est de nouveau aux «exotistes», aux universalistes. Robert Lahaise a raison, ce n'est que simple justice que de remettre en lumière ces écrivains longtemps méconnus.

1 Robert Lahaise, Guy Delahaye et la modernité littéraire, Montréal, Hurtubise/ HMH, 1987, 549 p.

2 Nous acceptons à contrecœur ce terme, «exotistes», de plus en plus répandu aujourd'hui, mais qui, à l'époque du conflit, fut presque exclusivement utilisé par Claude-Henri Grignon, un des adversaires les plus acharnés du groupe en question.

3 D'autres exemples du genre, tel celui de Saint-Denys Garneau, sautent à l'esprit. Louis Dantin, lui, suivra le cheminement contraire.

4 Dans l'Anthologie de Jules Fournier, Guy Delahaye avait donné l'impression qu'il s'agissait d'une revue en la classant avec l'Aube et en la désignant de la façon suivante: L'Encéphale (hors commerce).

5 L'article en question, du 10 août 1907, attaque le modernisme d'un poème de Guy Delahaye. Serait-ce par hasard le même A. Plouffe qui, en octobre 1909, sera élu conseiller du «Soc» estudiantin dont Lahaise est le président (p. 106)? Ou ce docteur Adrien Plouffe qu'on voit dans une photo d'amis canadiens prise dans sa chambre de l'Hôtel Jacob (p. 233) lors du séjour de Delahaye à Paris en 1912-1913? Signalons par ailleurs que Paul Morin avait déjà été l'objet d'une attaque semblable deux mois auparavant, de la part d'un nommé «Zoile», à laquelle il répond de façon fort spirituelle dans le Nationaliste du 2 juin 1907.

6 Renée défend le choix de «rimeur acrobatique» dans la parodie de Pauline alors que cette dernière, dans le même numéro, explique triomphalement à René Chopin que cette «erreur» provient en fait d'une correction apportée à son texte par la rédaction du journal.

7 Un examen attentif du Terroir de 1909, entre autres, montre que l'École littéraire est encore loin d'être «profondément terroiriste» et conserve son caractère plutôt éclectique. Le «triomphalisme de Dugas» au sujet de Delahaye n'a pas encore commencé en novembre 1909 et n'a donc pas pu incommoder les membres de l'École. Albert Laberge, qui aurait encouragé Delahaye à poser sa candidature, n'était présent à aucune des réunions où il était question de cette demande et il est donc possible que ce «La Haye», "Guy de Haize» ou «Guy de la Haize» (Procès-verbaux de l'École) était pour eux un parfait inconnu. Et qui voudrait introduire un jeune inconnu dans un groupe où la situation financière est telle que chacun s'inquiète de la part qu'il pourrait avoir à assumer dans les dettes encourues par la revue le Terroir? Quant au désir de Guy Delahaye d'en faire partie, il n'est peut-être pas si étonnant. Émile Nelligan en avait été membre, Albert Laberge l'était encore, la parution régulière d'une revue en faisait le groupe littéraire le plus visible et le plus actif à Montréal à cette époque...

8 Ce réveil sera d'ailleurs si spectaculaire que même des critiques européens comme Jacques Dubois seront portés comme exemple de la possibilité, pour une «littérature régionale», de devenir une littérature autonome et dynamique (l'Institution de la littérature: introduction à une sociologie, Bruxelles, Nathan/Labor, 1978, p. 136). 